\title{
Anisotropic spin dynamics of confined electrons in CdTe/ZnTe quantum structures
}

\author{
Shinichi Tomimoto, ${ }^{1}$ Shinsuke Nozawa, ${ }^{1}$ Yoshikazu Terai, ${ }^{2}, *$ Shinji Kuroda,${ }^{2}$ Kôki Takita, ${ }^{2}$ and Yasuaki Masumoto ${ }^{1}$ \\ ${ }^{1}$ Institute of Physics, University of Tsukuba, Tsukuba 305-8571, Japan \\ ${ }^{2}$ Institute of Materials Science, University of Tsukuba, Tsukuba 305-8573, Japan
}

(Received 22 November 2009; revised manuscript received 18 February 2010; published 12 March 2010)

\begin{abstract}
We have studied the spin dynamics of confined electrons in an ultrathin CdTe/ZnTe quantum well and self-assembled quantum dots by time-resolved Kerr-rotation technique. The dependence of the spin-precession frequency on the magnetic field direction shows the anisotropy of the $g$-factor tensor, which is the opposite of the usual quantum wells with moderate widths. The geometrical anisotropy of the confinement also affects the initial orientation of the electron spins created optically, as revealed clearly with the use of oblique-incidence pump pulses.
\end{abstract}

DOI: 10.1103/PhysRevB.81.125313

PACS number(s): 78.67.-n, 78.47.-p, 73.21.-b

\section{INTRODUCTION}

The spin physics of conduction electrons in semiconductors has received much interest especially in the lowdimensional structures such as quantum wells (QWs) and quantum dots (QDs). ${ }^{1}$ Some notable uniqueness of these structures comes from the finite momentum $(\boldsymbol{p} \neq 0)$ in the lowest-energy band states. In zinc-blende semiconductors, it leads to the splitting of the valence-band states with the total angular momentum $J=3 / 2$ between the heavy- and lighthole states. Heavy holes have projections of the angular momentum $J$ equal to $J_{z}= \pm 3 / 2$ while for light holes, they are $J_{z}= \pm 1 / 2$. Here the quantization axis $z$ is along the hole momentum $\boldsymbol{p}$, i.e., the direction of the confinement. Thus the geometrical anisotropy of the confinement structures affects the properties of these valence-band states, and make them essentially different from the corresponding atomic states with $J=3 / 2$. In thin QWs and in self-assembled QDs with small height-to-base ratio, the axis $z$ is along the crystalgrowth axis.

The anisotropy introduced to the valence-band states in this manner, in turn, influences on the properties of the conduction-band electrons. Here we note two effects on the electron spin $(S=1 / 2)$. One is the anisotropy of the electron $g$ factor. In thin QWs and QDs, the $g$ factor becomes anisotropic, and the component $g_{\|}=g_{z z}$ parallel to the growth axis is generally different from the perpendicular ones $g_{\perp}=g_{x x}=g_{y y}$. The first theory on this issue was given by Ivchenko and Kiselev. ${ }^{2}$ They presented a simple model allowing for the $\boldsymbol{k} \cdot \boldsymbol{p}$ mixing of the $\Gamma_{8}$ and $\Gamma_{7}$ states in the top valence bands into the $\Gamma_{6}$ state in the conduction band, and obtained a plain relationship between the $g$-factor anisotropy $\Delta g=g_{\perp}-g_{\|}$and the valence-band energy splitting. The idea is supported by some experiments which have been performed, for example, on GaAs/ $\mathrm{Al}_{x} \mathrm{Ga}_{1-x} \mathrm{As}$ (Refs. 3-5) and $\mathrm{CdTe} / \mathrm{Cd}_{1-x} \mathrm{Mg}_{x} \mathrm{Te}$ (Ref. 6) QWs.

The other effect on the electron spin is relevant to the optical connection between the conduction- and valenceband states, which governs the spin orientation of electrons created by circularly polarized light. ${ }^{7}$ If the system is isotropic, and there is no preferred axis for the projection of $\boldsymbol{J}$ of the holes, the electron-spin orientation is $50 \%$, and it is always along the direction of the excitation light. When the valence-band states are split with anisotropy, however, the situation is different. ${ }^{8}$ The spin orientation becomes a function of both the directions of the excitation light and the uniaxial anisotropy of the system. The respective roles of these directions will be made clear especially when they are not parallel.

In this paper, we report a comprehensive experimental study of the above-mentioned anisotropic spin dynamics of the confined electrons in an ultrathin CdTe/ZnTe QW and self-assembled QDs by time-resolved Kerr-rotation (TRKR) measurements. It reveals the anisotropy of the electron $g$ factor, which is discussed by comparison with a model calculation. This suggests an intrinsic feature to the ultrathin structures. The role of their geometrical anisotropy in the optical spin orientation is also studied with the use of oblique-incidence excitation pulses.

\section{EXPERIMENT}

In this study, we have used two samples of CdTe/ZnTe quantum structures grown by molecular-beam epitaxy. The detail of the fabrication procedure is described elsewhere. ${ }^{9}$ In both the samples, the growth of CdTe layers was performed by an alternate deposition of $\mathrm{Cd}$ and Te fluxes on $\mathrm{ZnTe}(100)$ surfaces. Under some appropriate conditions, 1/2 monolayer (ML) of CdTe is grown at each cycle of the alternate deposition in an autoregulated manner. In one sample, thus a 2-ML-thick CdTe layer was grown, and it was capped by a $\mathrm{ZnTe}$ layer with a thickness of $30 \mathrm{~nm}$. As this structure can be regarded as a $\mathrm{CdTe} / \mathrm{ZnTe}$ single $\mathrm{QW}$ with a well width of 2 ML, we will denote the sample by QW hereafter. In the other sample, a CdTe layer with a nominal thickness of 3.5 ML was grown by seven cycles of the above alternate deposition, and capped by ZnTe in the same way. As this amount of CdTe led to the growth of self-assembled QDs in the Stranski-Krastanow mode, we address the sample as QD below. Although the observation of the uncapped surface by an atomic force microscope showed that the typical size of the QDs was $20 \mathrm{~nm}$ in diameter and $2.7 \mathrm{~nm}$ in height, we guess the effective sizes of the carrier confinement were somewhat reduced by the $\mathrm{ZnTe}$ capping probably due to interdiffusion. This is suggested by the photoluminescence (PL) peak energy which is considerably higher than expected from the 


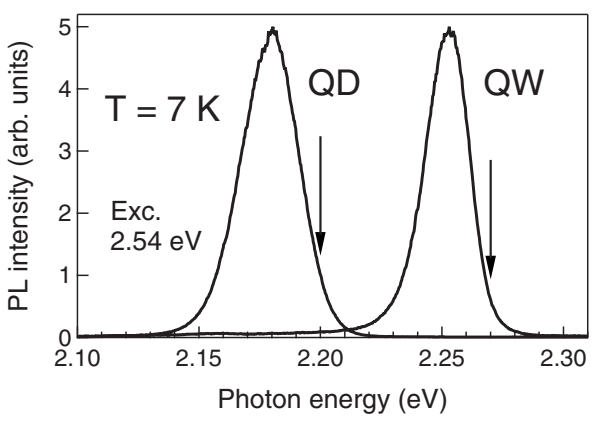

FIG. 1. PL spectra of the QD and the QW samples measured at a temperature $T=7 \mathrm{~K}$. The excitation photon energy is $E=2.54 \mathrm{eV}$. The arrows indicate the positions of 2.20 and $2.27 \mathrm{eV}$, which are the laser photon energies in most TRKR measurements for the QD and the QW, respectively.

above dot size. ${ }^{9}$ The areal density of the QDs was estimated to be $8 \times 10^{10} \mathrm{~cm}^{-2}$.

The spin dynamics is optically observed by the TRKR measurements. For the degenerate pump-probe experiment, an optical parametric oscillator pumped by a femtosecond mode-locked Ti:sapphire laser is used as a light source. It generates optical pulses with a duration of about $200 \mathrm{fs}$ at a repetition rate of $76 \mathrm{MHz}$. The central wavelength can be tuned from 525 to $665 \mathrm{~nm}$ (photon energy $E$ from 1.86 to $2.36 \mathrm{eV}$ ). This range covers the PL bands of the above two quantum structures (Fig. 1). The typical spectral width of the laser light is $\Delta E=13 \mathrm{meV}$. The laser output is separated into two beams, and one of them is used as a circularly polarized pump pulse which injects spin-polarized electron-hole pairs into the samples. The other is used as a probe pulse, which is spectrally filtered down to $\Delta E=5 \mathrm{meV}$. This is modulated between left and right circular polarizations at a frequency of $f=41.9 \mathrm{kHz}$ by a photoelastic modulator, and applied on the sample after a time delay. Then the reflected probe beam is directed to a Wollaston prism, and the two outputs of perpendicularly linear polarizations are detected by a balanced photoreceiver. In this setup, the polarization rotation $\Theta_{\mathrm{K}}$ of the reflected probe is measured by the amplitude of the $2 f(=83.8 \mathrm{kHz})$ component of the photoreceiver output ideally with no constant background. ${ }^{10,11}$ We have observed differential signal $\Delta \Theta_{\mathrm{K}}$ by modulating the pump intensity with an optical chopper at $210 \mathrm{~Hz}$ frequency. The overall time resolution of the system is about $0.4 \mathrm{ps}$.

In the measurements, the samples are mounted in a magneto-optical cryostat, containing a split-coil superconducting magnet for fields up to $10 \mathrm{~T}$. The field direction relative to the sample growth axis $z$ is either $\theta=90^{\circ}$ (Voigt geometry) or $45^{\circ}$. In the latter case, a small steering mirror is placed inside the cryostat just in front of the sample. ${ }^{4}$ The probe beam is always incident normal to the sample surface, i.e., parallel to the $z$ axis. This is also the case with the pump beam for the data presented in Secs. III A and III B. In Sec. III $\mathrm{C}$, however, the oblique-incidence pump beam is used. The experimental geometry in this case will be explained in detail in that section. The average powers of the pump and probe beams are always 7 and $1 \mathrm{~mW}$, and the typical spot diameters on the samples are $300 \mu \mathrm{m}$ and $200 \mu \mathrm{m}$, respectively.

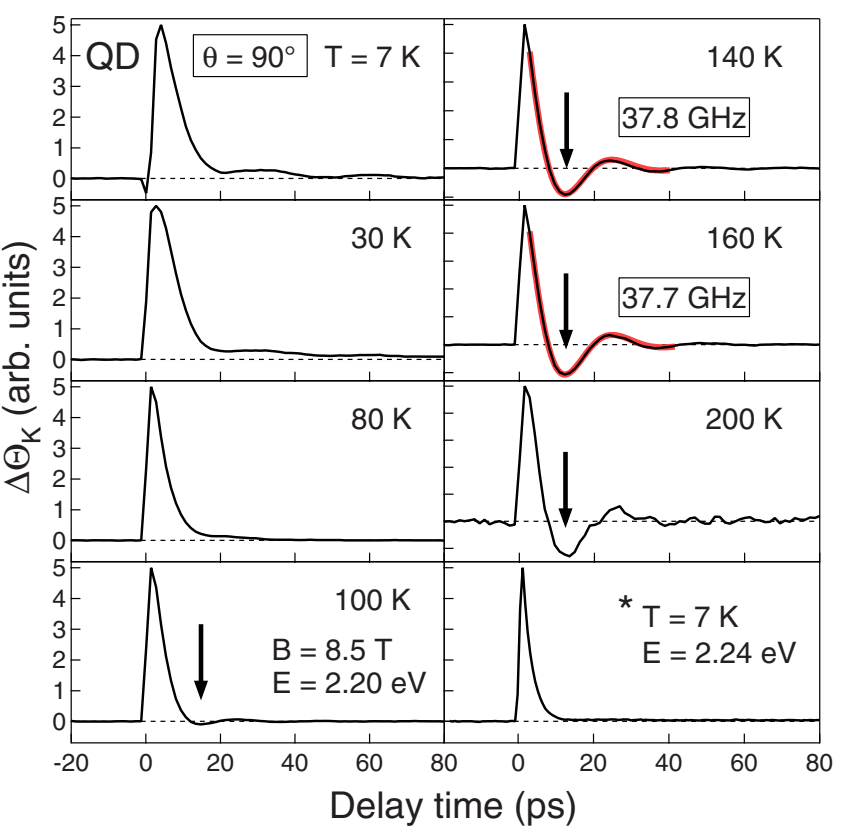

FIG. 2. (Color online) Time evolution of the Kerr rotation $\Delta \Theta_{\mathrm{K}}(t)$ of the QD sample observed at several temperatures indicated in each panel (magnetic field $B=8.5 \mathrm{~T}$, field direction $\theta=90^{\circ}$, and photon energy $E=2.20 \mathrm{eV}$ ). Thin lines show the experimental results. Thick lines $(T=140$ and $160 \mathrm{~K})$ show the fitting by Eq. (1), which gives the frequencies $\nu$ in rectangles. Arrows indicate the first oscillation bottoms. The data in the last panel denoted by an asterisk is the only one obtained at $E=2.24 \mathrm{eV}$ $(T=7 \mathrm{~K})$.

The PL spectra of the samples presented in this paper are obtained with an argon-ion laser (488.0 nm line, $2.54 \mathrm{eV}$ ) for excitation, and with a single-grating monochromator equipped with a liquid-nitrogen-cooled charge-coupled device for detection.

\section{RESULTS AND DISCUSSION}

\section{A. Thermal escape of holes and electron-spin precession}

Figure 2 shows the time evolution of the Kerr rotation $\Delta \Theta_{\mathrm{K}}(t)$ of the QD sample observed at the temperatures indicated in each panel. The magnetic field $B=8.5 \mathrm{~T}$ is applied perpendicular to the $z$ axis $\left(\theta=90^{\circ}\right)$. The photon energy of the pump and probe is $2.20 \mathrm{eV}$, which is at the high-energy side of the PL band (indicated by an arrow in Fig. 1). This is resonant to the heavy-hole exciton transition in the QDs, as will be verified in Sec. III C. At low temperatures below 100 $\mathrm{K}$, the main feature is simple decay within $20 \mathrm{ps}$. Although we can see weak long-lasting oscillation at the lowest temperature $(T=7 \mathrm{~K})$, the main decay component does not show the damped oscillation expected from the electron-spin precession below $100 \mathrm{~K}$. We suppose the weak oscillation is related to some kind of residual electrons which obtain spin coherence during the excitation. ${ }^{12}$

The lack of the oscillation associated with the main decay component is due to the coexistence of an electron and a heavy hole which are photocreated in a QD. In thin structures such as self-assembled QDs with small height-to-base 
ratio, heavy holes $\left(J_{z}= \pm 3 / 2\right)$ generally have negligibly small $g$-factor component perpendicular to the $z$ axis due to the split-off from light holes. ${ }^{13}$ Thus the heavy-hole spin can be assumed to be always along the $z$ axis, and it influences on the coexisting electron spin $\left(S_{z}= \pm 1 / 2\right)$ through the exchange interaction. In this situation, the quantum beat occurs between the radiative doublet $\left|S_{z}+J_{z}\right\rangle=| \pm 1\rangle$ and the nonradiative one $| \pm 2\rangle$ in the simplest picture. The beat amplitude depends on the field by the factor $(\omega / \Omega)^{2}$, where $\hbar \omega=g_{\perp} \mu_{\mathrm{B}} B$ is the electron Zeeman splitting $\left(\mu_{\mathrm{B}}\right.$ is the Bohr magneton), and $\hbar \Omega=\sqrt{(\hbar \omega)^{2}+\delta^{2}} \cdot{ }^{14,15}$ Here $\delta$ is the isotropic electron-hole exchange energy. Therefore, when the exchange energy is much larger than the electron Zeeman splitting $(\omega \ll \Omega)$, the quantum beat vanishes. It explains the lack of the oscillation below $T=100 \mathrm{~K}$.

As the temperature rises, the decay becomes faster and a different situation appears around $100 \mathrm{~K}$ : the negative overshoot denoted by arrows in Fig. 2. It evolves into the damped oscillation with large amplitude which we can see above 140 $\mathrm{K}$. This emergence of the damped oscillation is not a result of mere spectral redshift with temperature elevation. As the redshift of the PL peak reaches $0.04 \mathrm{eV}$ around $200 \mathrm{~K}$, we checked the $\Delta \Theta_{\mathrm{K}}(t)$ at the photon energy $E=2.24 \mathrm{eV}$ at $T$ $=7 \mathrm{~K}$. But no oscillation was observed (Fig. 2). The appearance of the oscillation at the high temperatures indicates the onset of the electron-spin precession, which, in turn, suggests the absence of holes from the QDs. Due to the very small valence-band offset of the common anion heterostructure, the thermal escape of holes from $\mathrm{CdTe} / \mathrm{ZnTe}$ quantum structures occurs very easily in comparison with electrons. Indeed, it is known as the main mechanism of the thermal quenching of the excitonic PL in CdTe/ZnTe QDs and QWs. ${ }^{16,17}$ In our QD sample, the PL intensity begins to decrease around $50 \mathrm{~K}$, and it is less than the maximum by almost 2 orders of magnitude at $T=100 \mathrm{~K} .{ }^{9}$ The temperature characteristic of the PL is consistent with the critical temperature of the onset of the electron-spin precession shown in Fig. 2. Above $100 \mathrm{~K}$, we can express the initial part of $\Delta \Theta_{\mathrm{K}}(t)$ by the following equation:

$$
\Delta \Theta_{\mathrm{K}}(t)=\Delta \Theta_{e l}(t)+\Delta \Theta_{e x c}(t) .
$$

The first term corresponds to the electron-spin precession,

$$
\Delta \Theta_{e l}(t)=I_{e l} \exp \left(-t / \tau_{e l}\right) \cos (2 \pi \nu t+\alpha) .
$$

The second term represents the relaxation of the excitonic spin polarization,

$$
\Delta \Theta_{\text {exc }}(t)=I_{\text {exc }} \exp \left(-t / \tau_{\text {exc }}\right),
$$

which will remain in part at any temperature, due to the inhomogeneity of the system. Assuming the initial phase $\alpha$ in Eq. (2) to be zero, we obtained the fitting shown as thick lines at $T=140$ and $160 \mathrm{~K}$ in Fig. 2. It gives the oscillation frequency $\nu$ shown in each panel with uncertainty of about $\pm 1 \%$.

Figure 3 shows $\Delta \Theta_{\mathrm{K}}(t)$ of the QW sample observed at several temperatures indicated in each panel $\left(B=4 \mathrm{~T}, \theta=90^{\circ}\right)$. The photon energy $2.27 \mathrm{eV}$ is in the high-energy side of the PL band (indicated by an arrow in Fig. 1), and this is resonant to the heavy-hole exciton transi-

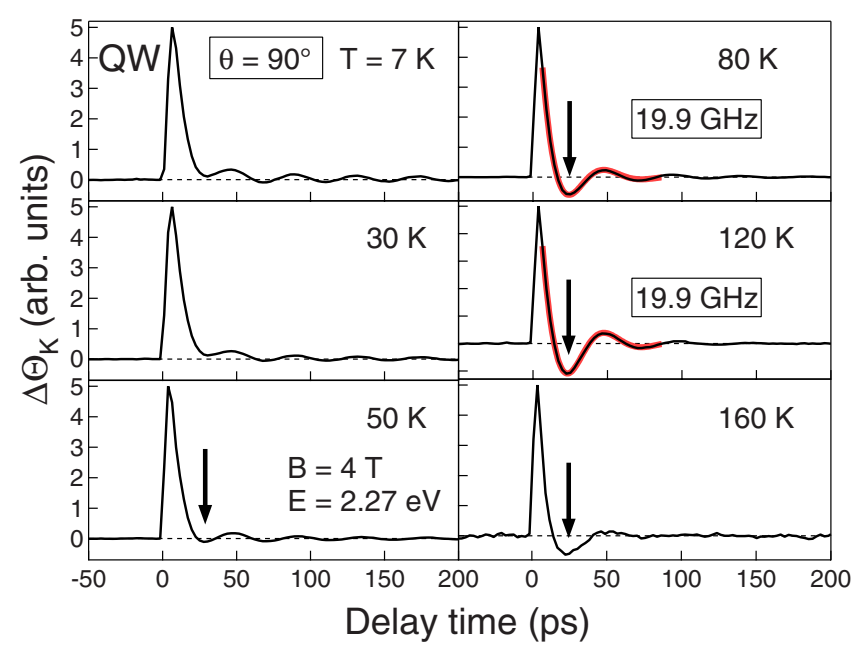

FIG. 3. (Color online) $\Delta \Theta_{\mathrm{K}}(t)$ of the QW sample observed at several temperatures indicated in each panel $(B=4 \mathrm{~T}, \theta$ $\left.=90^{\circ}, E=2.27 \mathrm{eV}\right)$. Thick lines $(T=80$ and $120 \mathrm{~K})$ show the fitting by Eq. (1), which gives the frequencies $\nu$ in rectangles.

tion of the QW, as will be mentioned in Sec. III C. The data shows very similar behavior to that of the QD. The main feature at low temperatures is the decay within $20 \mathrm{ps}$ and the weak long-lasting oscillation. ${ }^{12}$ The negative overshoot denoted by arrows in the figure appears around $T=50 \mathrm{~K}$, above which the initial part of $\Delta \Theta_{\mathrm{K}}(t)$ can be approximated by Eq. (1) including the exponentially decaying oscillation $\Delta \Theta_{e l}(t)$. The fitting is shown as thick lines at 80 and $120 \mathrm{~K}$ in Fig. 3. These features common to those of the QD are explained in the same way as in the preceding paragraph.

From these observations, we can estimate the lower and upper limits of the isotropic electron-hole exchange energy $\delta$. In the QD, for example, $\delta$ should be larger than the electron Zeeman splitting at $B=8.5 \mathrm{~T}, \hbar \omega=g_{\perp} \mu_{\mathrm{B}} B \simeq 160 \mu \mathrm{eV}$, to suppress the appearance of the quantum beat. It gives the lower limit of $\delta$. Meanwhile, as the electron and hole are separated thermally above $T=100 \mathrm{~K}$, the magnetic correlation $\delta$ should be smaller than the thermal energy $k_{\mathrm{B}} T=8.6 \mathrm{meV}$ at this critical temperature. It is the upper limit of $\delta$. In the QW, a similar range $0.083 \ll \delta<4.3 \mathrm{meV}$ is obtained by locating the critical temperature at $T=50 \mathrm{~K}$ in Fig. $3(B=4 \mathrm{~T})$. In the above estimation, we used the perpendicular $g$ factors $g_{\perp}$ which will be described in Sec. III B.

At present, the mechanism of the fast exciton spin relaxation (less than 20 ps in both the QD and the QW at $T=7 \mathrm{~K}$ ) is not clear. It may be dominated by the hole-spin depolarization. It is also possible that the inhomogeneity of the isotropic exchange $\delta$, on which the exciton beat frequency depends, affects the exciton signal decay. What we can state safely is that the signal lifetime is not limited by the recombination of excitons because the exciton PL lifetime exceeds $100 \mathrm{ps}$ at temperatures lower than $50 \mathrm{~K} .{ }^{9}$ The observation of the quantum beat between the bright exciton states $| \pm 1\rangle$ becomes difficult when the exciton signal decays so fast because their splitting by anisotropic exchange interaction is usually very small. ${ }^{23}$

As the temperature rises and comes close to $200 \mathrm{~K}$, the absolute signal intensity of $\Delta \Theta_{\mathrm{K}}(t)$ decreases, and the signal- 


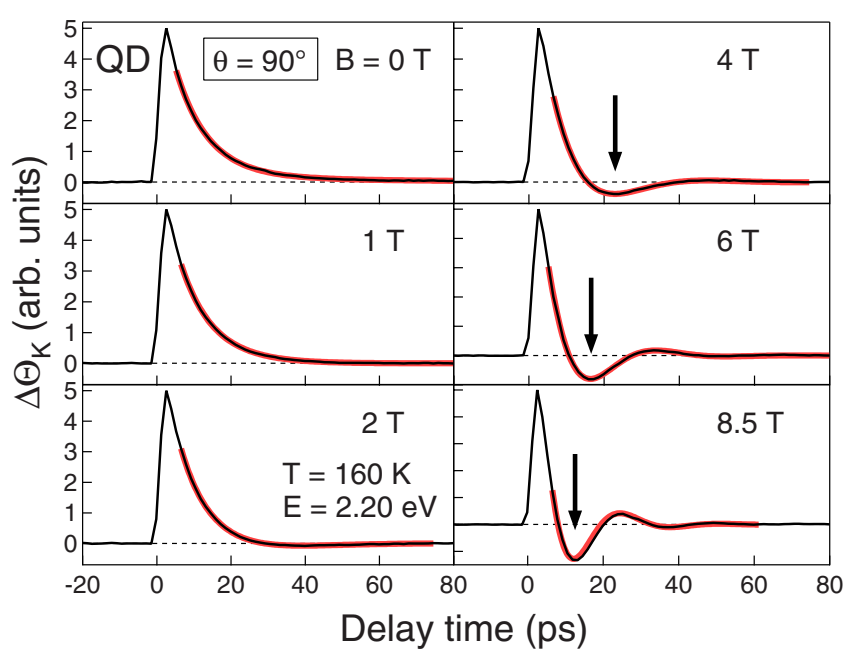

FIG. 4. (Color online) Magnetic field $(B)$ dependence of $\Delta \Theta_{\mathrm{K}}(t)$ of the QD sample at $T=160 \mathrm{~K}$. The field direction is perpendicular to the $z$ axis $\left(\theta=90^{\circ}\right)$. The photon energy of the pump and probe is $E=2.20 \mathrm{eV}$. The thin and thick lines show the experimental data and the fitting by Eq. (1), respectively. The first oscillation bottom indicated by arrows shifts to earlier times with increasing field.

to-noise ratio falls in both the QD and the QW samples. Consequently, the appropriate temperature range to observe the electron-spin precession independent of holes is narrow. We consider it is $130-170 \mathrm{~K}$ for the QD and $80-120 \mathrm{~K}$ for the QW sample. Within these ranges, the oscillation frequency $\nu$ scarcely depends on temperature. The values of $\nu$ obtained by the fitting are shown in Figs. 2 and 3 in rectangles. In the following sections, we focus on the electronspin dynamics within the above temperature ranges. ${ }^{18}$

\section{B. Electron $g$-factor anisotropy}

Figure 4 shows the magnetic field dependence of $\Delta \Theta_{\mathrm{K}}(t)$ of the QD sample $\left(\theta=90^{\circ}, T=160 \mathrm{~K}\right)$. The first oscillation bottom indicated by arrows shifts to earlier times with increasing field, which means larger frequency of the electronspin precession. The frequency $\nu$ is obtained by fitting the data with Eq. (1) (thick lines in Fig. 4), and it is plotted in Fig. 5(a) (filled circles) as a function of the field $B$. It shows linear dependence, $h \nu=g_{\perp} \mu_{\mathrm{B}} B$, shown as a solid line in the figure. From the gradient, we obtain the transverse $g$ factor $g_{\perp}=g\left(\theta=90^{\circ}\right)=-0.323$. The negative sign is revealed from the experiments under oblique-incidence optical pumping, which will be described afterward in Sec. III C. The precision of the $g$ factor thus obtained is comparable or better than $\pm 1 \% .{ }^{19}$ To know the component $g_{\|}=g_{z z}$ along the growth axis $z$, we have investigated the magnetic field dependence of $\Delta \Theta_{\mathrm{K}}(t)$ at the field direction $\theta=45^{\circ}$, as shown in Fig. 6. The thick lines in the figure are the fitting by Eq. (1). The electron part $\Delta \Theta_{e l}(t)$ is, however, replaced by

$$
\Delta \Theta_{e l}(t)=I_{e l} \exp \left(-t / \tau_{e l}\right)\{1+\cos (2 \pi \nu t)\}
$$

because the precession axis is now inclined from the $z$ axis by $45^{\circ}$. Then the obtained frequency $\nu$ is plotted in Fig. 5(a) as open squares. It is evident that they are lower than the

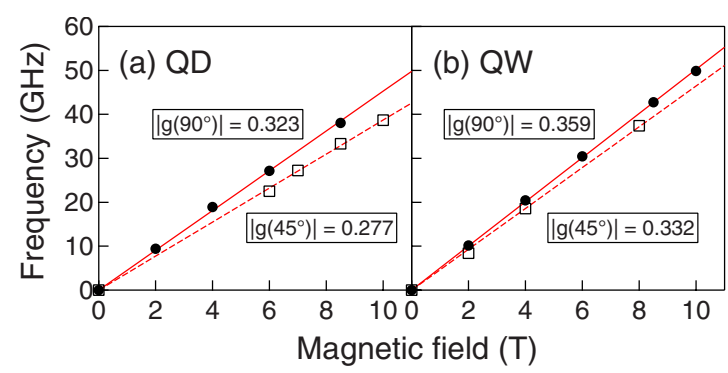

FIG. 5. (Color online) Magnetic field dependence of the spinprecession frequency of confined electrons in (a) QD and (b) QW. The filled circles show the experimental data at $\theta=90^{\circ}$ obtained from the fitting in Figs. 4 and 7. The open squares are the data at $\theta=45^{\circ}$ obtained from Figs. 6 and 8. The solid and broken lines give the $g$-factor values shown in rectangles at $\theta=90^{\circ}$ and $45^{\circ}$, respectively (Ref. 19).

frequencies at $\theta=90^{\circ}$, which manifests the anisotropy of the $g$ factor. The broken line in the figure gives $g\left(\theta=45^{\circ}\right) \equiv g_{45}=-0.277$. From this, we obtain the value of the parallel component $g_{\|}=-0.222$ by using the relation $g_{\|}^{2}=2 g_{45}^{2}-g_{\perp}^{2}, 3,4$

Figures 7 and 8 show the magnetic field dependence of $\Delta \Theta_{\mathrm{K}}(t)$ of the QW sample observed at the field directions $\theta=90^{\circ}$ and $45^{\circ}$, respectively. The same analysis gives the precession frequencies shown in Fig. 5(b) for the QW. Similar to the result in the $\mathrm{QD}$, the frequencies at $\theta=45^{\circ}$ are slightly lower than those at $\theta=90^{\circ}$. The solid and broken lines gives the values of $g_{\perp}=-0.359$ and $g_{45}=-0.332$, respectively. From these, we obtain the parallel component $g_{\|}=-0.303$. The values of the $g$-factor components, for both the QD and the QW, are summarized in Table I.

The most important feature of the observed $g$ factor is the fact that the parallel component is larger than the perpendicular one both in the QD and the QW $\left(g_{\|}>g_{\perp}\right)$. This is the

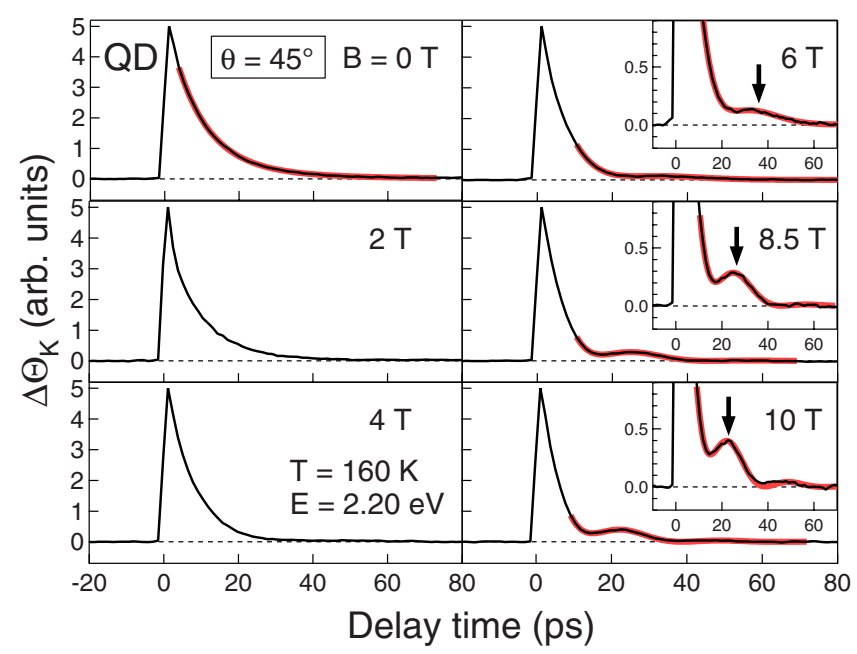

FIG. 6. (Color online) Magnetic field dependence of $\Delta \Theta_{\mathrm{K}}(t)$ of the QD sample at the field direction $\theta=45^{\circ}$. Insets right above in three panels show the closeups. The observed photon energy $(E=2.20 \mathrm{eV})$ and the sample temperature $(T=160 \mathrm{~K})$ are the same as those in Fig. 4. The thin and thick lines show the experimental data and the fitting by Eq. (1), respectively. The first oscillation peak denoted by arrows shifts to earlier times with increasing field. 


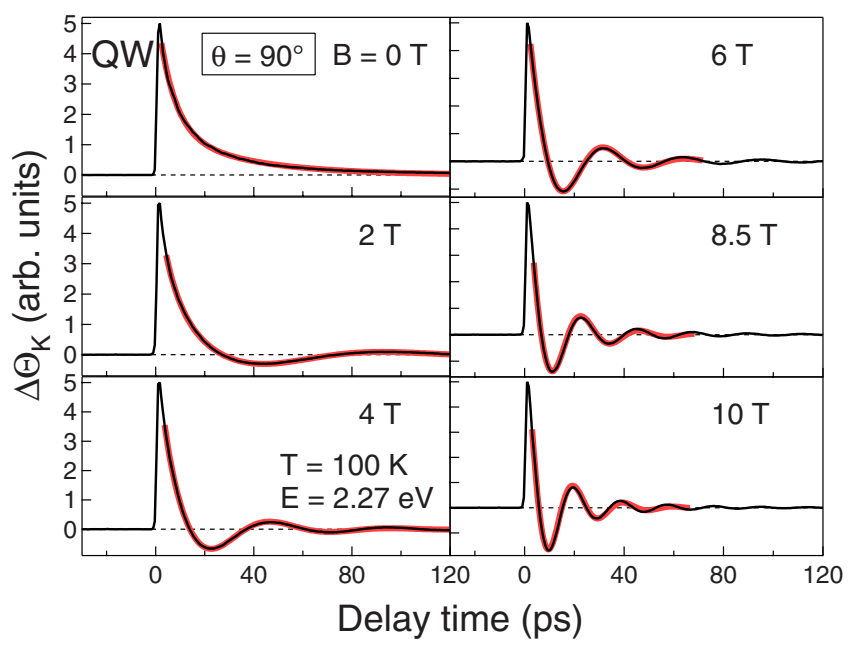

FIG. 7. (Color online) Magnetic field dependence of $\Delta \Theta_{\mathrm{K}}(t)$ of the QW sample at $\theta=90^{\circ}(T=100 \mathrm{~K}, E=2.27 \mathrm{eV})$.

marked difference from GaAs $/ \mathrm{Al}_{x} \mathrm{Ga}_{1-x}$ As (Refs. 3-5) and $\mathrm{CdTe} / \mathrm{Cd}_{1-x} \mathrm{Mg}_{x} \mathrm{Te}$ (Ref. 6) QWs where $g_{\|}$was reported smaller than $g_{\perp}$. This is different also from the claim of the $\boldsymbol{k} \cdot \boldsymbol{p}$ theory assuming infinitely high barriers in which the difference of the confinement energy in the valence-band states, larger for light holes than for heavy holes, inevitably leads to $g_{\|}<g_{\perp}{ }^{2}$ The experimental $g$ factors in Table I are closer to the bulk value of $\mathrm{ZnTe}(g=-0.4)$ than that of CdTe $(g=-1.6) .{ }^{20}$ This suggests that the penetration of the electron wave function into the ZnTe barrier has to be appropriately considered in the quantitative analysis. Yugova et al. discussed $g$-factor components in $\mathrm{GaAs} / \mathrm{Al}_{x} \mathrm{Ga}_{1-x}$ As QWs by their three-band Kane model..$^{5}$ This model is based on the theory by Kiselev et al., ${ }^{21}$ and is accounting for the $\boldsymbol{k} \cdot \boldsymbol{p}$ interaction between the lowest conduction band $\Gamma_{6}$ and the upper valence bands $\Gamma_{8}$ and $\Gamma_{7}$. This can deal with the $g$-factor anisotropy in the wide range of the confinement energy, even when it is close to the finite barrier height. It is profitable to try the calculation of the $g$ factors by this model for our case of $\mathrm{CdTe} / \mathrm{ZnTe}$ ultrathin structures and compare them with the experimental values.

In the calculation, we assume the one-dimensional rectangular potential well, and neglect the effect of the lateral confinement. We adopt band parameters without the influence of

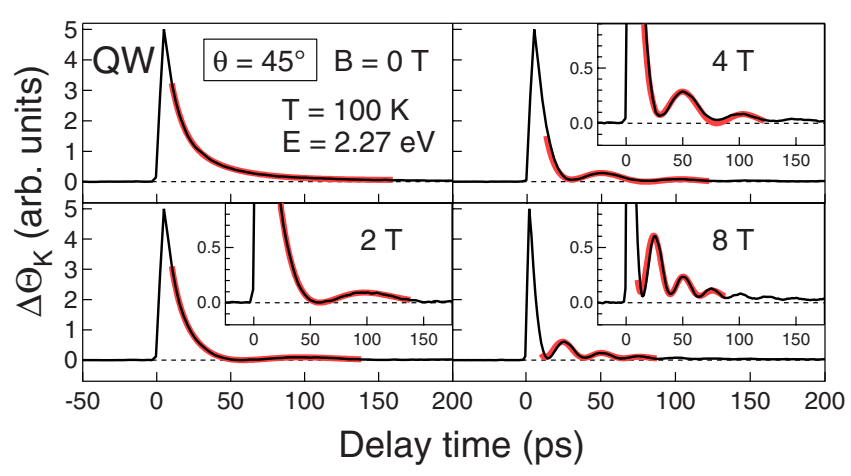

FIG. 8. (Color online) Magnetic field dependence of $\Delta \Theta_{\mathrm{K}}(t)$ of the QW sample at $\theta=45^{\circ}(T=100 \mathrm{~K}, E=2.27 \mathrm{eV})$. Insets right above in three panels show the closeups.
TABLE I. $g$-factor components and other parameters in the QD and the QW. The experimental values of $g_{\|}=g_{z z}$ and $g_{\perp}=g_{x x}=g_{y y}$ are compared with the calculated ones $g_{\|}^{\text {cal }}$ and $g_{\perp}^{\text {cal }}$, respectively. $E_{\mathrm{PL}}$ is the experimental PL peak energy of the QD at $T=160 \mathrm{~K}$ and of the QW at $100 \mathrm{~K} . d$ is the effective length of carrier confinement (potential-well width), which is determined so as to give the calculated PL energy $E_{\mathrm{PL}}^{c a l}$ close to the experimental values. $P_{c v}$ is the interband momentum matrix element.

\begin{tabular}{lcc}
\hline \hline Parameters & QD & QW \\
\hline$g_{\|}\left(g_{\|}^{c a l}\right)$ & $-0.222(-0.237)$ & $-0.303(-0.291)$ \\
$g_{\perp}\left(g_{\perp}^{c a l}\right)$ & $-0.323(-0.323)$ & $-0.359(-0.359)$ \\
$\Delta g=g_{\perp}-g_{\|}\left(\Delta g^{c a l}\right)$ & $-0.101(-0.086)$ & $-0.056(-0.068)$ \\
$E_{\mathrm{PL}}\left(E_{\mathrm{PL}}^{c a l}\right)(\mathrm{eV})$ & $2.15(2.14)$ & $2.24(2.24)$ \\
$d(\mathrm{~nm})$ & 0.5 & 0.32 \\
$2 P_{c v}^{2} / m_{0}$ in $\mathrm{CdTe}(\mathrm{eV})^{\mathrm{a}}$ & $22.7 \times 1.645$ & $22.7 \times 1.595$ \\
$2 P_{c v}^{2} / m_{0}$ in $\mathrm{ZnTe}(\mathrm{eV})$ & $25 \times 1.75$ & $25 \times 1.75$ \\
\hline \hline
\end{tabular}

${ }^{\mathrm{a}} m_{0}$ is the free-electron mass in vacuum.

strain for simplicity, ${ }^{22}$ which are explained in Appendix. Figure 9(a) shows the calculated result of the $g$-factor anisotropy $\Delta g=g_{\perp}-g_{\|}$as a function of the potential-well width $d$. For $d>1 \mathrm{~nm}, \Delta g$ is positive and has the maximum around $d=3 \mathrm{~nm}$. As $d$ increases from there, $\Delta g$ approaches asymptotically to zero, which is the bulk CdTe limit. In Fig. 9(b), the result of the same model for $\mathrm{GaAs} / \mathrm{Al}_{x} \mathrm{Ga}_{1-x} \mathrm{As}$ QWs $(x=0.35)$ is shown for comparison, in which we can see similar behavior. As $d$ decreases from $3 \mathrm{~nm}, \Delta g$ drops rapidly in both the two systems, and converges on zero at $d=0 \mathrm{~nm}$ (the bulk ZnTe or $\mathrm{Al}_{x} \mathrm{Ga}_{1-x} \mathrm{As}$ limit). The essential difference between the two systems appears at $d<1 \mathrm{~nm}$. Although $\Delta g$ in GaAs/ $\mathrm{Al}_{x} \mathrm{Ga}_{1-x}$ As QWs [Fig. 9(b)] is al-

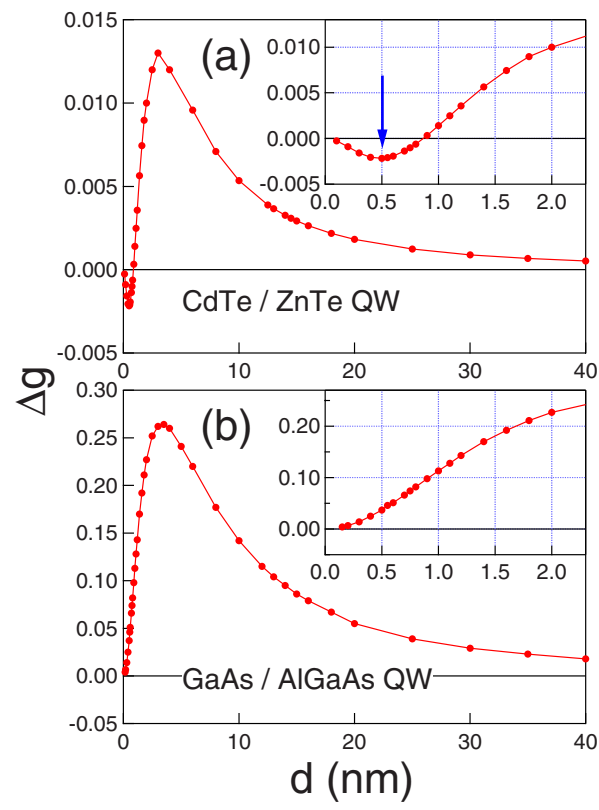

FIG. 9. (Color online) Calculated dependence of the $g$-factor anisotropy $\Delta g=g_{\perp}-g_{\|}$(filled circles) on the potential-well width $d$ for (a) CdTe/ZnTe QWs and for (b) GaAs/ $\mathrm{Al}_{x} \mathrm{Ga}_{1-x} \mathrm{As}$ QWs $(x=0.35)$. Insets present the closeups around $d=1 \mathrm{~nm}$. 
ways positive, there is a small negative region in $\mathrm{CdTe} / \mathrm{ZnTe}$ QWs as indicated by an arrow in the inset of Fig. 9(a). The experimental results of the negative $\Delta g\left(g_{\|}>g_{\perp}\right)$ in Table I should be attributed to the appearance of this region. From the results of Fig. 9, we suppose that the occurrence of the negative $\Delta g$ requires two conditions that may be satisfied in our samples: (1) an appropriate combination of the well and barrier materials and (2) an ultrathin potential well, i.e., a large electron confinement energy.

The sign of $\Delta g$ is uniquely determined by the numerical relativity of the following parameter $D$ in the well and barrier materials, ${ }^{5}$

$$
D=-\frac{P_{c v}^{2}}{3 m_{0}}\left(\frac{1}{E-V+E_{g}}-\frac{1}{E-V+E_{g}+\Delta_{S O}}\right),
$$

where $P_{c v}$ is the interband momentum matrix element, $m_{0}$ is the free-electron mass in vacuum, $E_{g}$ is the band-gap energy, and $\Delta_{S O}$ is the spin-orbit splitting in the valence band. $V$ is the potential energy for the conduction-band electron. When the eigen energy of the confined electron $E$ is given, the values of $D$ for the well $\left(D_{W}\right)$ and the barrier $\left(D_{B}\right)$ can be obtained from Eq. (5). Then, if $D_{W}>D_{B}\left(D_{W}<D_{B}\right), \Delta g$ becomes negative (positive). From this relation, we can expect the $g$-factor anisotropy, and can control it by the selection of materials and by tuning the eigenenergy $E$ of the confined state. We might be able to see the sign of $\Delta g$ change to positive, if we could investigate wider QWs. It is, however, difficult to prepare wide CdTe/ZnTe QW samples due to their relatively large lattice mismatch $(6.2 \%)$. [Please compare it with the values of GaAs/AlAs (0.1\%) (Ref. 5) and CdTe/ MgTe (1.0\%) (Ref. 6) heterostructures.] The deposition of 3.5 ML CdTe on ZnTe induces the formation of selfassembled QDs, whose dimensions are hard to control. ${ }^{9}$ In addition, the height of the QDs seems to be reduced unintentionally during the process of ZnTe capping on the CdTe layer due to interdiffusion. Therefore, it is difficult to perform experiments on wider QWs which were possible in the GaAs $/ \mathrm{Al}_{x} \mathrm{Ga}_{1-x} \mathrm{As}$ (Ref. 5) and CdTe/Cd $\mathrm{Cd}_{1-x} \mathrm{Mg}_{x} \mathrm{Te}$ (Ref. 6) QWs. In these systems, positive $\Delta g$ was certainly reported.

The calculation result in Fig. 9(a) presents rather smaller absolute values of $\Delta g$ (for example, $|\Delta g|=0.0022$ at $d=0.5 \mathrm{~nm}$ ) than the experimentally observed ones shown in Table I. The calculation of $\Delta g$, however, depends a lot on the values of $P_{c v}$. For Fig. 9(a), we used $2 P_{c v}^{2} / m_{0}=22.7 \mathrm{eV}$ for $\mathrm{CdTe}$ and $25 \mathrm{eV}$ for $\mathrm{ZnTe}$, which are given in a literature. ${ }^{7}$ We have to adopt a bit larger values of $2 P_{c v}^{2} / m_{0}$ to explain the experimental large $|\Delta g|$ by the model. We tried to find them, and the best choice is shown in Table I. When the values of $2 P_{c v}^{2} / m_{0}$ are larger than the literature ones by factors from 1.6 to 1.8 , the calculation gives $g_{\|}^{\text {cal }}$ and $g_{\perp}^{\text {cal }}$ for parallel and perpendicular $g$-factor components, respectively, shown in Table I. They agree fairly well with the experimental values. In this calculation, we used the potential-well width $d$ (the effective length of carrier confinement along the $z$ axis) determined so as to give the lowest optical transition energies $E_{\mathrm{PL}}^{c a l}$ close to the experimental PL peaks $E_{\mathrm{PL}}$ for the respective samples (Table I). The small widths $d$ might arise from the interdiffusion during the ZnTe capping.
The discrepancy of $2 P_{c v}^{2} / m_{0}$ between the values in Table I and in the literature may indicate the limit of availability of the model to the ultrathin structures. As this model is developed originally for $\mathrm{GaAs} / \mathrm{Al}_{x} \mathrm{Ga}_{1-x} \mathrm{As}$ QWs with widths larger than $4 \mathrm{~nm}$, there are some difficulties to apply it directly to our thin structures below $1 \mathrm{~nm}$. We think the following two problems can be pointed out. First, the model does not take account of the strain effects for simplicity. The strain should be present in our thin systems, and may be important for electron $g$ factors because it affects the energy splitting of the valence-band states. Second, in the model, the electronic state is represented by the usual Bloch functions with periodic parts kept unchanged from the ones in bulk materials. This may not be appropriate for our thin systems. As $P_{c v}$ depends on the periodic part, it may be effectively different in the ultrathin structures from the bulk value. We allowed the QD and the QW to have slightly different values of $2 P_{c v}^{2} / m_{0}$ in CdTe as shown in Table I.

At present, we know no alternative model more satisfying. Despite the above problems, the current model is rather simple and can reproduce the feature of experimental results qualitatively. The problems, especially in the quantitative analysis, will be solved by more sophisticated theory and are left for the future.

\section{Spin orientation under oblique-incidence optical pumping}

In Sec. III B, we discussed the $g$-factor components accepting that they have negative signs. We describe here the experimental determination of the sign by the TRKR measurement. One of the standard methods to determine the sign is based on the interaction between the electron and polarized nuclear spins, which uses the known sign of the hyperfine coupling constant as a clue. ${ }^{4,23}$ It is, however, difficult to apply the method to our samples because we could not observe any signatures of the nuclear-spin polarization in the electron-spin dynamics, even in the oblique magnetic field $\left(\theta=45^{\circ}\right)$, although our measurement has been done without the temporal modulation of the pump circular polarization. This might be attributed to the rather fast electron-spin relaxation in our samples. Another method for the determination of the $g$-factor sign is to discern the direction of the electron-spin precession around the magnetic field. This can be done by making a finite angle between the initial orientation of the photocreated electron spin and the spinobservation axis in the plane perpendicular to the magnetic field. The basis of this method was developed in the PL spectroscopy. ${ }^{24,25}$ Here we apply it to the TRKR measurements.

Figure 10(a) shows the configuration of the sample, magnetic field direction, and light beams in our experiment. The magnetic field is perpendicular to the $z$ axis $\left(\theta=90^{\circ}\right)$, and both the pump and probe beams lie in the plane normal to the field. But the incidence angle of the pump beam is $\phi_{0}=45^{\circ}$, which is the only point different from the measurements mentioned so far. This will generally lead to the deflection of the initial spin orientation from the $z$ axis toward the pump beam axis as depicted in the figure. Then the expectation value of the electron spin $\langle\boldsymbol{S}\rangle$ begins to precess around the field following the equation 

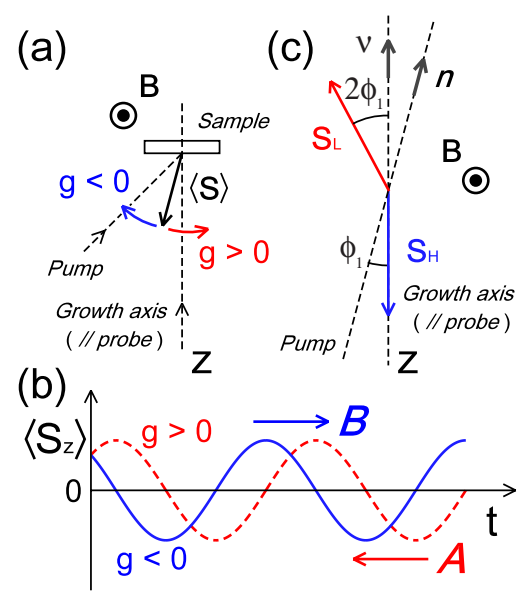

FIG. 10. (Color online) Schematic to explain the experiment with oblique-incidence optical pumping. (a) Configuration of the sample, magnetic field direction, and light beams. (b) Time evolution of the TRKR signal $\Delta \Theta_{\mathrm{K}}(t) \propto\left\langle S_{z}\right\rangle$ for two cases with opposite $g$-factor signs. (c) Mean spins of electrons excited from heavy-hole states, $S_{\mathrm{H}}$, and from light-hole states, $S_{\mathrm{L}}$. Details are mentioned in the text.

$$
\frac{d\langle\boldsymbol{S}\rangle}{d t}=\boldsymbol{\Omega} \times\langle\boldsymbol{S}\rangle-\frac{\langle\boldsymbol{S}\rangle}{\tau_{e l}},
$$

where $\boldsymbol{\Omega}=g \mu \boldsymbol{B} / \hbar$ is the Larmor frequency. Thus the direction of the precession depends on the sign of the $g$ factor as expressed in Fig. 10(a). The probe beam remains parallel to the $z$ axis. Therefore it is still the spin observation axis. In this configuration, the time evolution of the TRKR signal $\Delta \Theta_{\mathrm{K}}(t) \propto\left\langle S_{z}\right\rangle$ will depend on the $g$-factor sign. The solid and broken curves in Fig. 10(b) present the expected TRKR signals in cases of negative and positive $g$ factors, respectively. There appears a phase difference between the two curves if the initial spin orientation is tilted from the $z$ axis.

In the practical experiment, we have compared $\Delta \Theta_{\mathrm{K}}(t)$ at two opposite magnetic field directions. If the $g$ factor is positive, $\Delta \Theta_{\mathrm{K}}(t)$ will be observed as the solid curve in Fig. 10(b) when the magnetic field is inverted from that shown in Fig. 10(a). Then the phase shift of the oscillation indicated as the arrow A in Fig. 10(b) will arise along with the field inversion. If the $g$ factor is negative, the phase shift will be opposite (the arrow B). Therefore we can determine the $g$-factor sign by observing the phase-shift direction when the field is inverted.

Figure 11 shows the experimental results in the QD sample. The respective panels from (a) to (e) include two experimental data of $\Delta \Theta_{\mathrm{K}}(t)$ at $B=+8.5 \mathrm{~T}$ (thin solid lines) and $-8.5 \mathrm{~T}$ (thin broken lines), which are taken at the different photon energies $E$ from 2.18 to $2.26 \mathrm{eV}(T$ $\left.=140 \mathrm{~K}, \phi_{0}=45^{\circ}\right)$. We define the positive magnetic field as having the direction shown in Fig. 10(a). The two data are fitted by Eq. (1), and the initial phases $\alpha$ in Eq. (2) are determined. The thick curves with light tones in Fig. 11 are the fitting ones, which determine the initial phases $\alpha_{+}$and $\alpha_{-}$ for $B=+8.5 \mathrm{~T}$ and $-8.5 \mathrm{~T}$, respectively. At (a) $E=2.18$ and (b) $2.20 \mathrm{eV}$, there is little difference between the two data at

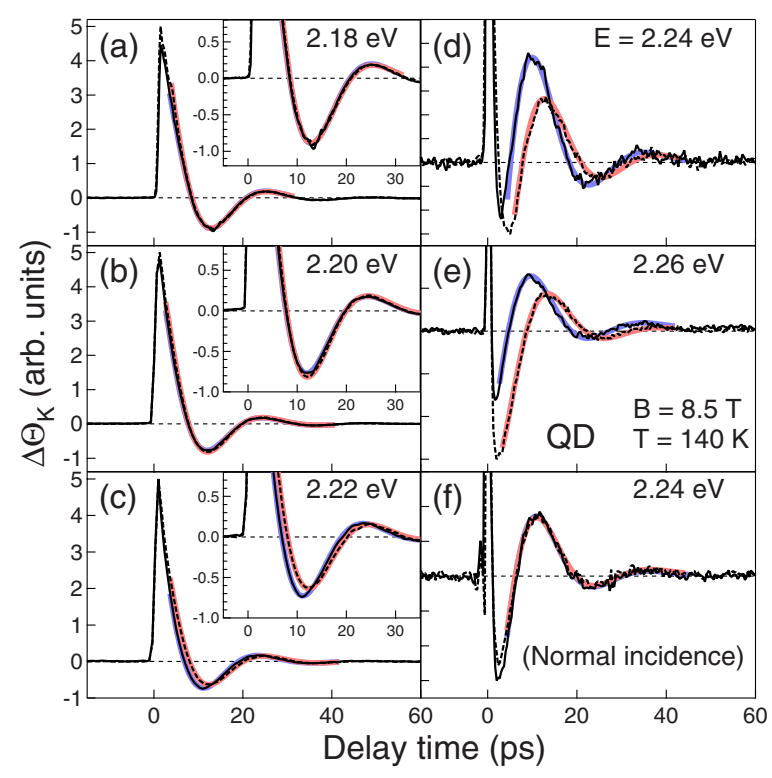

FIG. 11. (Color online) $\Delta \Theta_{\mathrm{K}}(t)$ of the QD sample at the pump incidence angle $\phi_{0}=45^{\circ}(T=140 \mathrm{~K})$. Each panel includes two experimental data for $B=+8.5 \mathrm{~T}$ (thin solid lines) and $-8.5 \mathrm{~T}$ (thin broken lines) observed at the indicated photon energy $E$. Thick curves with light tones are the fitting by Eq. (1). Insets in (a)-(c) are the closeups around the first oscillation bottoms. Only the data in (f) are observed at the normal pump incidence $\phi_{0}=0^{\circ}$ to be compared with those in (d). The two data at $B= \pm 8.5 \mathrm{~T}$ are almost overlapping in (f).

$B= \pm 8.5$ T. But a small phase difference appears at (c) 2.22 $\mathrm{eV}$, where the broken line oscillates a little behind the solid one. This phase difference increases rapidly between 2.22 and (d) $2.24 \mathrm{eV}$. The observed large difference at $2.24 \mathrm{eV}$ does not appear when the pump incidence is normal to the sample surface $\left(\phi_{0}=0^{\circ}\right)$ as shown in Fig. 11(f). In Fig. 12(a), the phase shift defined as $\psi=\left(\alpha_{+}-\alpha_{-}\right) / 2$ is plotted as a function of the photon energy $E$. The value $\psi$ corresponds to the deflection angle of the initial spin orientation from the $z$ axis. It reaches $\psi=27.5^{\circ}$ at $E=2.26 \mathrm{eV}$. All the data in Figs. 11(c)-11(e) exhibit the phase delay along with the magnetic field inversion from positive to negative which can be represented as the arrow B in Fig. 10(b). This indicates that the perpendicular $g$-factor component $\left[g_{\perp}=g\left(\theta=90^{\circ}\right)\right]$ is negative. As the difference between $g_{\perp}$ and $g_{45}=g\left(\theta=45^{\circ}\right)$ is not very large (Fig. 5), we assumed the parallel component $g_{\|}$ also to be negative.

Figure 13 shows the experimental results in the QW sample at four photon energies from 2.27 to $2.31 \mathrm{eV}$ $\left(T=100 \mathrm{~K}, \phi_{0}=45^{\circ}\right)$. The two data of $\Delta \Theta_{\mathrm{K}}(t)$ in each panel are obtained at the field $B=+6 \mathrm{~T}$ (thin solid lines) and $-6 \mathrm{~T}$ (thin broken lines). They are fitted by Eq. (1) (thick curves with light tones). The observed behavior is very similar to that of the QD sample. The phase difference between the two data of $\Delta \Theta_{\mathrm{K}}(t)$ is small at (a) $E=2.27$ and (b) $2.28 \mathrm{eV}$ but it can be recognized that the broken line oscillates a little behind the solid one, just as in the QD sample. This confirms the negative $g$ factor. The phase shift increases sharply between 2.28 and (c) $2.29 \mathrm{eV}$. The $\psi$ obtained from the fitting is plotted against the photon energy in Fig. 12(b). 


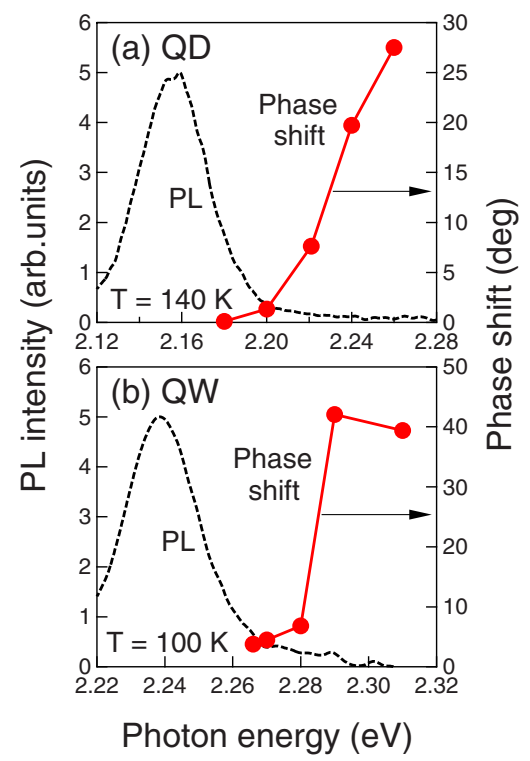

FIG. 12. (Color online) Photon-energy dependence of the phase shift $\psi=\left(\alpha_{+}-\alpha_{-}\right) / 2$ for (a) the QD $(T=140 \mathrm{~K})$ and (b) the QW $(100 \mathrm{~K})$. PL spectra of the samples obtained at the same temperatures are shown as broken lines.

In both the QD and the QW, there is some critical energy $E_{c}$ for the appearance of the large phase shift, i.e., the occurrence of the initial spin orientation tilted from the $z$ axis. It is between 2.22 and $2.24 \mathrm{eV}$ for the QD, and between 2.28 and $2.29 \mathrm{eV}$ for the QW. In Fig. 12, PL spectra of the samples are also presented as broken lines to make clear the relative location of $E_{c}$. It should be noted that the spectra are obtained at the same temperatures as those in the TRKR measurements (Figs. 11 and 13) for the respective samples. The PL intensities are smaller than those at $T=7 \mathrm{~K}$ (Fig. 1) by about 2 orders of magnitude. The critical energy $E_{c}$ is about 70 meV higher than the PL peak in the QD and about $50 \mathrm{meV}$ higher in the QW. The existence of $E_{c}$ is attributed to the

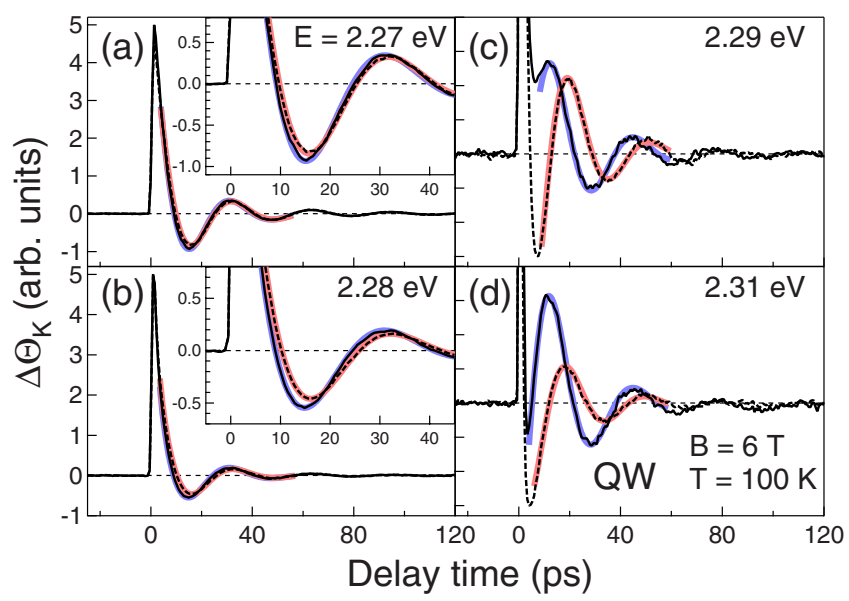

FIG. 13. (Color online) $\Delta \Theta_{\mathrm{K}}(t)$ of the QW sample at the pump incidence angle $\phi_{0}=45^{\circ}(T=100 \mathrm{~K})$. Each panel includes two experimental data for $B=+6 \mathrm{~T}$ (thin solid lines) and $-6 \mathrm{~T}$ (thin broken lines) observed at the indicated photon energy $E$. Thick curves with light tones are the fitting by Eq. (1). Insets in (a) and (b) are the closeups around the first oscillation bottoms. energy splitting of the heavy- and light-hole states in these quantum structures.

Here we consider the situation represented by Fig. 10(c). The pump beam propagates along the direction of the unit vector $\boldsymbol{n}$ in the sample crystal, which is tilted from the $z$ axis by an angle $\phi_{1}$. For this general case of an arbitrary pump direction, the respective mean spins of electrons excited from $J_{z}= \pm 3 / 2$ states (heavy holes), $S_{\mathrm{H}}$, and from $J_{z}= \pm 1 / 2$ states (light holes), $S_{\mathrm{L}}$, were calculated by D'yakonov and Perel', ${ }^{8}$

$$
\begin{gathered}
S_{\mathrm{H}}=\frac{-\boldsymbol{\nu}(\boldsymbol{\nu} \cdot \boldsymbol{n})}{1+(\boldsymbol{\nu} \cdot \boldsymbol{n})^{2}}, \\
S_{\mathrm{L}}=\frac{3 \boldsymbol{\nu}(\boldsymbol{\nu} \cdot \boldsymbol{n})-2 \boldsymbol{n}}{5-3(\boldsymbol{\nu} \cdot \boldsymbol{n})^{2}},
\end{gathered}
$$

where $\boldsymbol{\nu}$ is the unit vector along the $z$ axis [Fig. 10(c)]. They assumed the dipole transition in a circularly polarized light field. The vectors $S_{\mathrm{H}}$ and $S_{\mathrm{L}}$ are depicted in the figure. Although $S_{\mathrm{L}}$ is deflected away from the $z$ axis by an angle, approximately $2 \phi_{1}, S_{\mathrm{H}}$ is stuck to the $z$ axis. This difference between the two initial states of the optical transition (heavy and light holes) is important to understand the experimental data in Figs. 11-13. The inflexibility of the direction of $\boldsymbol{S}_{\mathrm{H}}$ is related to the fact that the expectation values of the perpendicular components $\left\langle J_{x}\right\rangle$ and $\left\langle J_{y}\right\rangle$ are always zero in any states consisting of $\left|J_{z}= \pm 3 / 2\right\rangle$, which is not the case for $\left|J_{z}= \pm 1 / 2\right\rangle$.

In the actual experiment, the angle $\phi_{1}$ is rather small due to the refraction at the sample surface, although the pump incidence angle is large $\left(\phi_{0}=45^{\circ}\right) . \phi_{1}$ is found to be about $13.6^{\circ}$ from the refraction index of $\mathrm{ZnTe}(\sim 3)$ around the experimental photon energy. ${ }^{26,27}$ Then the product $\boldsymbol{\nu} \cdot \boldsymbol{n}$ in Eqs. (7) and (8) can be regarded as 1 for approximation. From this, we can readily see that the mean spin $\langle\boldsymbol{S}\rangle$ would be parallel to the pump beam axis $\boldsymbol{n}$, if the probability ratio between the two optical transitions from heavy- and lighthole states were 3 to 1 , as in the bulk materials. This would lead to the phase shift $\psi$ equal to $\phi_{1} \sim 13.6^{\circ}$. In our QD and QW samples, however, $\psi$ varies with the laser photon energy $E$ from nearly zero to the values much larger than $\phi_{1}$, as seen in Fig. 12. The mean spin $\langle\boldsymbol{S}\rangle$ is not necessarily parallel to the excitation light beam.

The dependence of $\psi$ on $E$ comes from the energy splitting of the heavy- and light-hole states. In both the samples, the observed $\psi$ is very small at low photon energies around the PL band (Fig. 12), where the pump is resonant to the transition from heavy-hole states. Then the deflection of $\langle\boldsymbol{S}\rangle$ from the $z$ axis vanishes as expected from Eq. (7). As the photon energy rises, the contribution of the transition from light-hole states increases. This is reflected in the rise of $\psi$. Above the critical energy $E_{c}$, the values of $\psi$ are significantly larger than $\phi_{1} \sim 13.6^{\circ}$, which indicates the dominance of the light-hole transition. The slower rise of $\psi$ in the QD [Fig. 12(a)] than in the QW [Fig. 12(b)] is attributed to the larger inhomogeneous broadening of the transition energy in the ensemble QDs which is suggested by the width of the PL band. [Note that the horizontal scale in Fig. 12(a) is larger 
than that in Fig. 12(b).] In both Figs. 11 and 13, we notice that the inversion of the signal oscillation from cosine to minus cosine occurs just at the critical energy $E_{c}$. As the pump and probe energies are degenerate in our experiment, it is probably due to the change in the probe resonance to the light-hole transition.

The result in Fig. 12 is intriguing because it indicates that we can obtain unprecedented flexibility of the orientation of the initialized electron spins by using the light-hole transition and by tuning the pump incidence angle.

\section{SUMMARY}

We have studied the spin dynamics of photocreated electrons confined in an ultrathin CdTe/ZnTe quantum well and self-assembled quantum dots by time-resolved Kerr-rotation technique. The spin-precession frequency depends on the magnetic field direction, which shows the anisotropy of the $g$-factor tensor, $\Delta g=g_{\perp}-g_{\|} \neq 0$, arisen from the geometry of the carrier confinement. The observed anisotropy $\Delta g<0$ is the opposite of the usual quantum wells with moderate widths. It is discussed with a model calculation, and a guideline is provided to design systems with desired $\Delta g$. The geometrical anisotropy of the confinement also affects the initial orientation of the photoelectron spins, as revealed from the use of oblique-incidence optical pumping. The orientation is stuck to the $z$ axis in the case of excitation from heavy-hole states. Directional flexibility can, however, be allowed to a large extent by using the excitation from lighthole states.

\section{ACKNOWLEDGMENTS}

This work was supported by Grant-in-Aid for Scientific Research No. 20244044 from the MEXT of Japan and R\&D promotion scheme funding international joint research promoted by NICT of Japan.
TABLE II. Fundamental band-structure parameters of CdTe and ZnTe used in the model calculation in Sec. III B. $m_{\text {bulk }}$ and $m_{\text {hh }}$ are the electron- and heavy-hole effective masses, respectively. $g_{\text {bulk }}$ is the electron $g$ factor in the bulk materials.

\begin{tabular}{lcc}
\hline \hline Parameters & CdTe & ZnTe \\
\hline$E_{g}(\mathrm{eV})$ & 1.59 & 2.39 \\
$\Delta_{S O}(\mathrm{eV})$ & 0.93 & 0.91 \\
$m_{\text {bulk }} / m_{0}$ & 0.099 & 0.122 \\
$m_{\mathrm{hh}} / m_{0}$ & 0.4 & 0.6 \\
$g_{\text {bulk }}$ & -1.6 & -0.4 \\
\hline \hline
\end{tabular}

\section{APPENDIX: BAND PARAMETERS}

Here we summarize the band parameters of CdTe and ZnTe which are used in the model calculation described in Sec. III B (Refs. 20 and 22). Table II presents fundamental values which are used in the calculation of Fig. 9(a) as they are. The offsets in the conduction and valence bands are $\Delta E_{C}=0.69 \mathrm{eV}$ and $\Delta E_{V}=0.11 \mathrm{eV}$, respectively. In Ref. 6, two different values of $2 P_{c v}^{2} / m_{0}$ in $\mathrm{ZnTe}(29.2$ and $20.7 \mathrm{eV})$ are on a list. So it is assumed tentatively to be $25 \mathrm{eV}$ in the calculation of Fig. 9(a). For the calculation of the values shown in parentheses of Table I, we considered the thermal effect on the band structures because they are compared with the experimental $g$ factors obtained at rather high temperatures $(T=160 \mathrm{~K}$ in the QD and $100 \mathrm{~K}$ in the QW). Then the temperature gradients $-3.58 \times 10^{-4}$ and $-4.49 \times 10^{-4} \mathrm{eV} / \mathrm{K}$ are assumed for CdTe and $\mathrm{ZnTe}$ band gaps, respectively. The ratio of the offsets $\Delta E_{C} / \Delta E_{V}$ is treated as independent of temperature. All the parameters used for the calculation of Fig. 9(b) $\left(\mathrm{GaAs} / \mathrm{Al}_{x} \mathrm{Ga}_{1-x} \mathrm{As} \mathrm{QWs}, x=0.35\right)$ are the same as those in Ref. 5.
* Present address: Division of Materials and Manufacturing Science, Graduate School of Engineering, Osaka University, Suita 5650871, Japan.

${ }^{1}$ Spin Physics in Semiconductors, edited by M. I. Dyakonov (Springer, Berlin, 2008).

${ }^{2}$ E. L. Ivchenko and A. A. Kiselev, Sov. Phys. Semicond. 26, 827 (1992).

${ }^{3}$ P. Le Jeune, D. Robart, X. Marie, T. Amand, M. Brousseau, J. Barrau, V. Kalevich, and D. Rodichev, Semicond. Sci. Technol. 12, 380 (1997)

${ }^{4}$ A. Malinowski and R. T. Harley, Phys. Rev. B 62, 2051 (2000).

${ }^{5}$ I. A. Yugova, A. Greilich, D. R. Yakovlev, A. A. Kiselev, M. Bayer, V. V. Petrov, Yu. K. Dolgikh, D. Reuter, and A. D. Wieck, Phys. Rev. B 75, 245302 (2007).

${ }^{6}$ A. A. Sirenko, T. Ruf, M. Cardona, D. R. Yakovlev, W. Ossau, A. Waag, and G. Landwehr, Phys. Rev. B 56, 2114 (1997).

${ }^{7}$ Optical Orientation, edited by F. Meier and B. P. Zakharchenya (North-Holland, Amsterdam, 1984).
${ }^{8}$ M. I. D'yakonov and V. I. Perel', Sov. Phys. Semicond. 7, 1551 (1974).

${ }^{9}$ Y. Terai, S. Kuroda, K. Takita, T. Okuno, and Y. Masumoto, Appl. Phys. Lett. 73, 3757 (1998).

${ }^{10}$ K. Sato, H. Hongu, H. Ikekame, Y. Tosaka, M. Watanabe, K. Takanashi, and H. Fujimori, Jpn. J. Appl. Phys. 32, 989 (1993).

${ }^{11}$ A. Kanno and Y. Masumoto, Phys. Rev. B 73, 073309 (2006).

${ }^{12}$ The weak long-lasting oscillation at low temperature is seen both in the QD (Fig. 2) and the QW (Fig. 3). The origin of this feature is not clear until now. As the oscillation amplitude relative to the main exciton component is rather small, and it strongly depends on the location on the sample surface, we suspect it is from extrinsic origins, such as defect-bound electrons which obtain spin coherence by the pump irradiation. The corresponding perpendicular $g$ factors $\left(\left|g_{\perp}\right|=0.30\right.$ and 0.43 for the QD and the QW, respectively) differ from those obtained for optically generated electrons (Fig. 5).

${ }^{13}$ B. Kuhn-Heinrich, W. Ossau, E. Bangert, A. Waag, and G. Land- 
wehr, Solid State Commun. 91, 413 (1994).

${ }^{14}$ T. Amand, X. Marie, P. Le Jeune, M. Brousseau, D. Robart, J. Barrau, and R. Planel, Phys. Rev. Lett. 78, 1355 (1997).

${ }^{15}$ M. Dyakonov, X. Marie, T. Amand, P. Le Jeune, D. Robart, M. Brousseau, and J. Barrau, Phys. Rev. B 56, 10412 (1997).

${ }^{16}$ F. Tinjod, K. Kheng, J. Bleuse, and H. Mariette, Physica E 17, 68 (2003).

${ }^{17}$ F. Tinjod, S. Moehl, K. Kheng, B. Gilles, and H. Mariette, J. Appl. Phys. 95, 102 (2004).

${ }^{18}$ In Sec. III B, we describe the electron-spin dynamics in the QD and the QW samples at temperatures of 160 and $100 \mathrm{~K}$, respectively. Even at these temperatures, the exciton signal is significant. The exciton fraction in the signal $P_{e x c}=I_{e x c} /\left(I_{e x c}+I_{e l}\right)$ is estimated to be 0.39 and 0.72 in the QD and the QW samples at the respective temperatures. These values seem to approach unity as the temperature decreases because the negative overshoot denoted by arrows in Figs. 2 and 3 disappears. At the low temperatures, however, the confident determination of $P_{e x c}$ is difficult because of the existence of the small oscillation from extrinsic origins (Ref. 12).

${ }^{19}$ At high magnetic fields, where the oscillation signal is relatively clear, the frequency can be determined rather uniquely by careful fitting with uncertainty of about $\pm 1 \%$. This uncertainty is smaller than the size of symbols in Fig. 5. As the $g$ factors are based on several data points thus obtained, their precision would be comparable or better than $\pm 1 \%$. Meanwhile, the uncertainty of the frequency values at low fields become larger. For example, at $B=2 \mathrm{~T}$, it is estimated to be about $\pm 11 \%$ in the QD sample $\left(\theta=90^{\circ}\right)$. It is, however, still comparable with the size of the symbols in Fig. 5 because the frequency itself becomes small at the low fields. Therefore error bars are omitted in the figure.

${ }^{20}$ Numerical Data and Functional Relationships in Science and Technology, Landolt-Börnstein, New Series, Group III Vol. 17b, Pt. 22a, edited by O. Madelung (Springer, Berlin, 1982).

${ }^{21}$ A. A. Kiselev, K. W. Kim, and E. L. Ivchenko, Phys. Status Solidi B 215, 235 (1999).

${ }^{22}$ C. G. Van de Walle, Phys. Rev. B 39, 1871 (1989).

${ }^{23}$ I. A. Yugova, A. Greilich, E. A. Zhukov, D. R. Yakovlev, M. Bayer, D. Reuter, and A. D. Wieck, Phys. Rev. B 75, 195325 (2007).

${ }^{24}$ V. K. Kalevich, B. P. Zakharchenya, K. V. Kavokin, A. V. Petrov, P. Le Jeune, X. Marie, D. Robart, T. Amand, J. Barrau, and M. Brousseau, Phys. Solid State 39, 681 (1997).

${ }^{25}$ V. K. Kalevich, E. L. Ivchenko, A. Yu Shiryaev, M. M. Afanasiev, A. Yu Egorov, M. Ikezawa, and Y. Masumoto, Semicond. Sci. Technol. 23, 114008 (2008).

${ }^{26}$ H. H. Li, J. Phys. Chem. Ref. Data 13, 103 (1984).

${ }^{27}$ K. Sato and S. Adachi, J. Appl. Phys. 73, 926 (1993). 